\title{
Efectos de la desnutrición sobre la colagenización de anastomosis intestinales: análisis de procolágeno y telopéptido carboxiterminal mediante radioinmunoanálisis
}

\author{
J. M. Álamo, A. Galindo, S. Morales, G. Dazaํ, M. Socas, G. Suárez-Artacho, J. M. Suárez-Grau, J. García- \\ Moreno, F. Pareja y M. A. Gómez
}

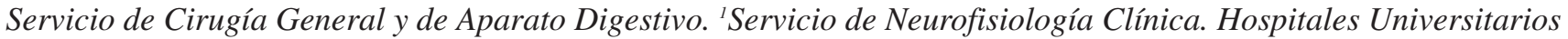
Virgen del Rocío. Sevilla

\section{RESUMEN}

Introducción: diversos factores influyen en la cicatrización correcta de las suturas intestinales tras la práctica de una resección intestinal. Uno de los factores más implicados es el estado nutricional del paciente.

Objetivos: evaluar la influencia de la desnutrición inducida sobre la viabilidad de una anastomosis intestinal primaria mediante el análisis del procolágeno (PINP) como marcador de la síntesis de colágeno I, y del telopéptido carboxiterminal del colágeno I (ICTP) como marcador de la destrucción del mismo.

Métodos: 40 ratas Wistar y material de radioinmunoensayo. Métodos: diseñamos 2 grupos de ratas, 20 animales por cada grupo: grupo control (A) y grupo "desnutrición" (B). Se analiza PINP e ICTP mediante RIA sobre tejido colónico homogeneizado, preanastomótico y anastomótico.

Resultados: existen unos niveles menores de PINP en el colon de las ratas del grupo B comparado con el colon del grupo A $(0,3620$ y $0,4340 \mu \mathrm{g} / \mathrm{g}$ respectivamente) $(\mathrm{p}=0,032)$. Hay un mayor nivel de ICTP analizado en el colon del grupo B $(0,9545$ en contraposición a 0,8460 $\mathrm{gg} / \mathrm{g}$ en el grupo A) $(p=0,875)$. En las anastomosis del grupo B existe una menor síntesis de PINP en comparación con el grupo A $(0,376$ y $0,468 \mu \mathrm{g} / \mathrm{g}$ respectivamente, $p=0,002$ ).

Conclusiones: la anastomosis colónica incrementa los niveles de PINP e ICTP en el tejido cicatricial ( $p=0,000)$; la malnutrición reduce la colagenización de las anastomosis $(p=0,000)$.

Palabras clave: Anastomosis intestinal. Procolágeno. Telopéptido carboxiterminal. Albúmina.

\begin{abstract}
Introduction: some clinical, anatomo-pathological, and technical factors influence the correct healing of intestinal suture following an intestinal resection. One of the most influential factors is patient nutritional status.

Objectives: to evaluate the influence of malnutrition on the viability of primary intestinal anastomosis by the analysis of collagen I deposition.

Methods: 40 Wistar rats, radioimmunoassay material. We used 2 groups of rats, 20 animals in each group: a control group (A) and a "malnutrition" group (B).

Results: there was a decrease in PINP (procollagen) deposition in the colon of group $\mathrm{B}$ rats as compared to the colon of group $\mathrm{A}$ $(0.3620$ and $0.4340 \mu \mathrm{g} / \mathrm{g}$ respectively) $(\mathrm{p}=0.032)$. There is an increase in ICTP (carboxyterminal telopeptide) in the colon of group B $(0.9545$ as against $0.8460 \mu \mathrm{g} / \mathrm{g}$ in group $\mathrm{A})(\mathrm{p}=0.875)$. In anastomoses of group B there was a decrease in PINP synthesis as compared to group $\mathrm{A}(0.376$ and $0.468 \mathrm{mg} / \mathrm{g}$ respectively, $\mathrm{p}=0.002)$. As regards ICTP, there was an increase in group $B(p=0.330)$. In relation to the control group no differences were observed in ICTP increases in group $B(p=1)$.

Conclusions: colonic anastomosis increases the levels of PINP and ICTP in healed tissue $(p=0.000)$; malnutrition reduces collagenization in anastomoses $(p=0.000)$.
\end{abstract}

Key words: Intestinal anastomosis. Procollagen. Telopeptide. Albumin.

Álamo JM, Galindo A, Morales S, Daza G, Socas M, Suárez-Artacho G, Suárez-Grau JM, García-Moreno J, Pareja F, Gómez MA. Efectos de la desnutrición sobre la colagenización de anastomosis intestinales: análisis de procolágeno y telopéptido carboxiterminal mediante radioinmunoanálisis. Rev Esp Enferm Dig 2007; 99: 76-83.

Recibido: 14-12-05.

Aceptado: 27-10-06.

Correspondencia: José María Álamo Martínez. C/ José Loquillo 27, Bq. 3, $7^{\circ} \mathrm{C} .41003$ Sevilla. e-mail: jmalamom@ terra.es

\section{INTRODUCCIÓN}

Aunque parece claro que factores como la presencia de una peritonitis o un mal estado clínico del paciente pueden hacer que la cicatrización de una sutura intestinal no sea del todo favorable, existen otros factores en los que no está tan claro su papel en la viabilidad de una sutura intestinal. Uno de estos factores es la desnutrición. En 
este trabajo intentamos evaluar la influencia que la desnutrición inducida presenta sobre el metabolismo del colágeno en las anastomosis intestinales. Para ello utilizamos, tal como hacen Bode y Mosorin (1) y Bode y Karttunen (2), el procolágeno (PINP) y el telopéptido carboxiterminal (ICTP) como marcadores, respectivamente, de la síntesis y de la destrucción del colágeno I en el tejido colónico.

El colágeno (el predominante en el adulto es el tipo I) constituye el principal componente del tejido conectivo fibroso, membranas basales, cartílago, córnea, válvulas cardiacas y otros tejidos especializados. La unidad fundamental del colágeno es el tropocolágeno. En el interior del fibroblasto, la molécula de procolágeno es aún soluble debido a que posee un fragmento carboxiterminal polipeptídico extra, que pierde en el espacio extracelular por la acción de peptidasas procolágenas. Este procolágeno, en el caso del colágeno I es denominado PINP, sustancia que utilizaremos en este modelo experimental como marcador de la síntesis de colágeno. El telopéptido carboxiterminal unido por enlaces trivalentes es liberado por metaloproteinasas durante su degradación. Es este telopéptido del colágeno tipo I (ICTP) la otra molécula que analizaremos en el tejido colónico como marcador de la destrucción del colágeno.

La hipótesis de trabajo es que la desnutrición favorece el fallo de las suturas intestinales. El objetivo de este estudio está encaminado a evaluar cómo este supuesto influye en la viabilidad de las anastomosis intestinales mediante el análisis del colágeno depositado en la anastomosis.

\section{MATERIAL Y MÉTODOS}

El material utilizado a tal fin es el siguiente: fármacos anestésicos (ketamina y xilacina), material quirúrgico e hilos de polipropileno, de lactomer y de seda, balanza de precisión, criotubos y nitrógeno líquido para el procesado y almacenaje de muestras titulares, tubos Z serum Sep Clot Activator y centrifugadora para el procesado y almacenaje de muestras sanguíneas, material para el procesado de muestras titulares (nitrógeno líquido, pulverizador, tampón PBS/Tween-20, tampón de lavado de péptido C, balanza de precisión, agitador de vidrio y centrífuga) y material para el radioinmunoanálisis: kits comerciales de radioinmunoensayo "ICTP-RIA ${ }^{\circledR}$ e "Intact PINP ${ }^{\circledR} "$, micropipetas, tubos de test, papel absorbente, agua destilada y contador-gamma.

Hemos diseñado un modelo experimental animal de desnutrición inducida con ratas Wistar en el que utilizamos 2 grupos de ratas:

1. Grupo control (A): 20 ratas sanas, bien nutridas y mantenidas de forma adecuada.

2. Grupo "desnutrición" (B): 20 ratas sanas, sometidas a ayuno, excepto agua, durante 72 horas. Para comprobar el estado de desnutrición se analizan la pérdida de peso, la concentración plasmática de albúmina previa y posterior al ayuno (mediante extracción sanguínea por punción aórtica) y al análisis cualitativo de la grasa mesentérica (objetivación “de visu”).

Cada grupo, como hemos comentado, consta de 20 sujetos a estudio, muestra que hemos considerado suficiente para obtener datos significativos después de analizar los primeros datos del grupo preliminar y del grupo control. Todos los modelos experimentales han sido validados desde el punto de vista estadístico.

La anestesia del animal se ha realizado mediante inyección intraperitoneal de ketamina y xilacina. Se practicó una resección colónica y una anastomosis primaria término-terminal con sutura sintética a cada animal, extirpando el tejido anastomótico al cuarto día para analizar posteriormente el comportamiento del colágeno tanto en el "colon sano" recogido en la primera intervención como en el "colon anastomótico" recogido en la segunda. El sacrificio se ha realizado mediante inyección intracardiaca de cloruro potásico.

Para analizar las modificaciones producidas en el depósito de colágeno nos basamos en la detección en el tejido anastomótico de ICTP y PINP. Para el análisis radioinmunológico de las muestras, y debido a que estas sustancias se detectan en la práctica clínica habitual en fluídos biológicos como el plasma o la orina, hemos preparado las muestras recogidas de forma conveniente. Para ello, y siguiendo la metodología recomendada, hemos pulverizado y homogeneizado mediante métodos mecánicos y tripsinización, el tejido recogido con el fin de poder analizarlo en la gammacámara. Previamente, y con las primeras muestras, hemos elaborado una curva de radioactividad para facilitar la lectura de muestras posteriores.

Los resultados recogidos se han analizado de forma estadística, realizándose en primer lugar estadística descriptiva de las variables cuantitativas. Para comparar dichas variables, según los grupos de estudio, hemos utilizado como pruebas de significación estadística para datos apareados la t de Student para muestras independientes y ANOVA con corrección de Bonferroni para medidas repetidas. Se ha establecido el nivel de significación estadística en $\mathrm{p}<0,05$.

$\mathrm{El}$ presente proyecto ha sido respetuoso con lo dispuesto por la normativa legal vigente y, en particular, por el Real Decreto 223/1988, sobre "Protección de los animales utilizados para experimentación y otros fines científicos”.

\section{RESULTADOS}

Para validar el grupo B (desnutrición) hemos utilizado 3 parámetros: peso del animal, concentración plasmática de albúmina y variación cualitativa de la grasa mesentérica. Los resultados conseguidos son: a) peso del animal en ayunas del $93 \%$ del peso inicial: reducción de peso de 350,25 g de media en el grupo control a 325,75 g ( $\mathrm{p}=0,032)$; $\mathrm{b})$ disminución de la concentración plasmática de albúmina al 81,57\%: de $982 \mathrm{mg} / \mathrm{dl}$ de media de albuminemia en el grupo control a $801 \mathrm{mg} / \mathrm{dl}$ en el grupo B (p = 0,000) (Fig. 1); y 
c) disminución en la cantidad de grasa mesentérica en todas las ratas del grupo B en comparación con el grupo control $(\mathrm{p}=0,000)$. Esta observación es meramente cualitativa y "de visu", pudiendo objetivarse una reducción en el grosor del mesenterio de las ratas desnutridas.

Una vez comprobada la validez de los modelos experimentales utilizados, pasamos a evaluar el comportamiento del colágeno en el tejido colónico antes y después de las anastomosis en cuanto a concentraciones tisulares $(\mu \mathrm{g} / \mathrm{g})$ de PINP e ICTP (Tablas I y II). En la figura 2 se representan las medias de los niveles encontrados de PINP e ICTP obtenidos en cada grupo de estudio, observándose cómo existe un mayor depósito de PINP y unas concentraciones menores de ICTP en las ratas del grupo control respecto a los animales del grupo B. Desglosamos a continuación los datos obtenidos.

En las muestras titulares de colon recogido previo a realizar la anastomosis podemos observar unos niveles de PINP en las ratas del grupo B menores que en el grupo control $(0,3620$ y $0,4340 \mu \mathrm{g} / \mathrm{g}$ respectivamente), dato estadísticamente significativo $(\mathrm{p}=0,032)$. Asimismo, existe una tendencia a depositarse en mayor cuantía el ICTP analizado en el colon de los animales del grupo B respecto al grupo A $(0,9545$ frente a $0,8460 \mu \mathrm{g} / \mathrm{g}$ en el grupo control) $(\mathrm{p}=0,875)$ (Fig. 3).

Comparando las concentraciones tisulares de PINP como dato aislado en el tejido anastomótico de ambos grupos, se objetiva que en las anastomosis del grupo B también existe unos niveles menores en su síntesis respecto al grupo control A (0,376 y $0,468 \mu \mathrm{g} / \mathrm{g}$ respectivamente) $(\mathrm{p}=$ 0,002). En cuanto a os datos analizados de ICTP (Fig. 4), no se observan diferencias significativas $(\mathrm{p}=0,330)$.

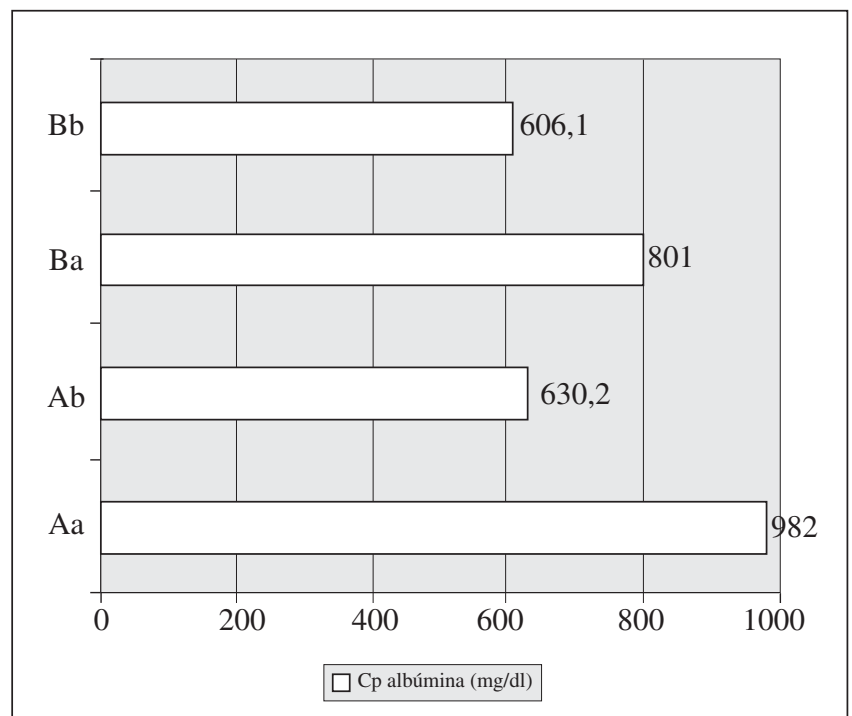

Fig. 1. Diferencias en la albuminemia de las ratas de los grupos $A$ y $B$ (Aa: previo a la anastomosis en grupo $A$; $A b$ : tras la realización de anastomosis colónica en grupo $\mathrm{A}$; $\mathrm{Ba}$ : previo a la anastomosis en grupo $B$; Bb: tras la realización de anastomosis colónica en grupo B; Cp: concentración plasmática).
Tabla I. Concentraciones plasmáticas de PINP $(\mu \mathrm{g} / \mathrm{g})$

\begin{tabular}{lcccc}
\hline & $A a$ & $A b$ & $B a$ & $B b$ \\
\hline 1 & 0,48 & 0,49 & 0,43 & 0,44 \\
2 & 0,52 & 0,53 & 0,41 & 0,44 \\
3 & 0,41 & 0,42 & 0,42 & 0,42 \\
4 & 0,42 & 0,47 & 0,44 & 0,45 \\
5 & 0,39 & 0,48 & 0,48 & 0,51 \\
6 & 0,34 & 0,39 & 0,24 & 0,30 \\
7 & 0,49 & 0,53 & 0,27 & 0,29 \\
8 & 0,39 & 0,47 & 0,22 & 0,23 \\
9 & 0,44 & 0,46 & 0,45 & 0,46 \\
10 & 0,47 & 0,46 & 0,36 & 0,38 \\
11 & 0,48 & 0,54 & 0,30 & 0,31 \\
12 & 0,51 & 0,55 & 0,42 & 0,43 \\
13 & 0,44 & 0,47 & 0,37 & 0,39 \\
14 & 0,31 & 0,33 & 0,43 & 0,45 \\
15 & 0,49 & 0,50 & 0,42 & 0,43 \\
16 & 0,36 & 0,38 & 0,34 & 0,37 \\
17 & 0,52 & 0,58 & 0,39 & 0,27 \\
18 & 0,44 & 0,48 & 0,25 & 0,27 \\
19 & 0,39 & 0,43 & 0,33 & 0,37 \\
20 & 0,39 & 0,44 & 0,27 & 0,31 \\
Media & 0,434 & 0,468 & 0,362 & 0,376 \\
Mediana & 0,44 & 0,47 & 0,38 & 0,385 \\
Rango & $0,34-0,52$ & $0,33-0,58$ & $0,22-0,48$ & $0,23-0,51$ \\
\hline a: tejido colónico previo a anastomosis; b: tejido anastomótico. &
\end{tabular}

Tabla II. Concentraciones plasmáticas de ICTP $(\mu \mathrm{g} / \mathrm{g})$

\begin{tabular}{lcccc}
\hline & $A a$ & $A b$ & $B a$ & $B b$ \\
\hline 1 & 0,41 & 1,31 & 0,74 & 2,41 \\
2 & 0,83 & 1,25 & 0,84 & 2,16 \\
3 & 1,09 & 1,75 & 1,04 & 1,74 \\
4 & 0,88 & 1,15 & 1,04 & 2,03 \\
5 & 0,71 & 1,16 & 0,99 & 1,32 \\
6 & 0,94 & 1,61 & 0,32 & 1,12 \\
7 & 0,50 & 1,28 & 0,86 & 0,75 \\
8 & 0,43 & 1,57 & 0,95 & 2,03 \\
9 & 1,06 & 1,17 & 1,59 & 1,57 \\
10 & 0,76 & 1,60 & 1,05 & 2,17 \\
11 & 0,97 & 1,32 & 1,18 & 1,71 \\
12 & 1,04 & 1,49 & 1,03 & 0,76 \\
13 & 1,01 & 1,25 & 0,84 & 0,94 \\
14 & 1,26 & 1,56 & 0,70 & 1,20 \\
15 & 0,99 & 2,14 & 0,81 & 2,23 \\
16 & 1,08 & 1,42 & 1,26 & 1,76 \\
17 & 1,03 & 0,71 & 0,86 & 1,54 \\
18 & 0,28 & 0,82 & 0,85 & 1,80 \\
19 & 0,96 & 1,75 & 0,98 & 2,21 \\
20 & 0,69 & 2,19 & 1,16 & 1,35 \\
Media & 0,846 & 1,425 & 0,954 & 1,640 \\
Mediana & 0,95 & 1,37 & 0,965 & 1,725 \\
Rango & $0,28-1,26$ & $0,71-2,19$ & $0,32-1,26$ & $0,75-2,23$ \\
\hline a: tejido colónico previo a anastomosis; b: tejido anastomótico. &
\end{tabular}

a: tejido colónico previo a anastomosis; b: tejido anastomótico. 


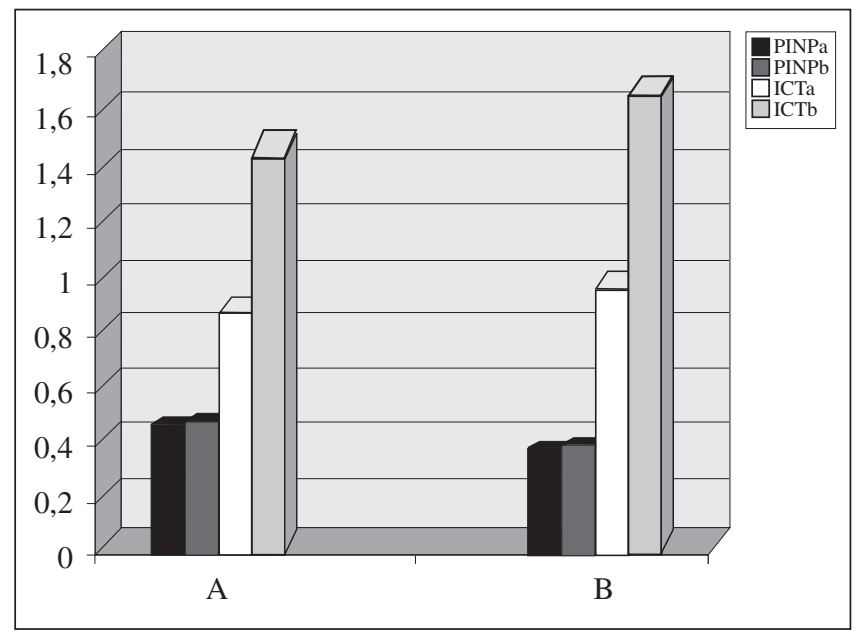

Fig. 2. Medias de PINP e ITCP $(\mu \mathrm{g} / \mathrm{g})$ por grupos de estudio A y B (a: tejido colónico previo a anastomosis; b: colon anastomótico).



Fig. 3. Media de PINP e ICTP $(\mu \mathrm{g} / \mathrm{g})$ analizado en el colon previo a la anastomosis de cada grupo de estudio.

Comparando los niveles de PINP depositado en el tejido colónico antes y después de la anastomosis puede objetivarse que en el grupo control existe un incremento de PINP al cuarto día de la anastomosis de un 7,83\% (0,034 $\mu \mathrm{g} / \mathrm{g})(\mathrm{p}=0,000)$. En el grupo B, este aumento es tan sólo del $3,87 \%(\mathrm{p}=0,086)$. No obstante, comparando estos incrementos con el grupo control A, el aumento de PINP en la anastomosis en el grupo B carece de significación alguna ( $\mathrm{p}=0,356)$ (Fig. 5).

Pese a no establecerse como objetivo inicial la observación de incidencia de dehiscencias de sutura por ser este un parámetro sujeto a la técnica realizada, hemos observado que mientras que no se han apreciado dehiscencias de sutura ni complicaciones en el grupo control, hemos objetivado dos fugas anastomóticas en el grupo B. Un animal del grupo B sufrió además una evisceración parcial en el cuarto día del postoperatorio.



Fig. 4. Media de PINP e ICT $(\mu \mathrm{g} / \mathrm{g})$ en el tejido anastomótico de cada grupo.

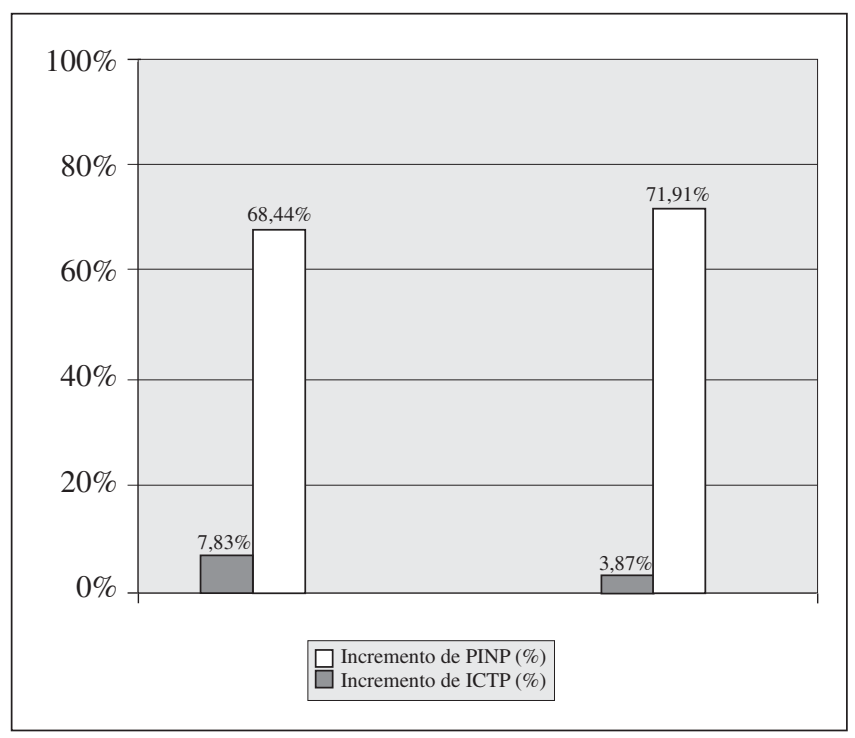

Fig. 5. Incremento de PINP e ICTP (\%) en el tejido anastomótico al $4^{\circ}$ día de la anastomosis.

\section{DISCUSIÓN}

Numerosos autores han evaluado la calidad de la sutura intestinal realizada tras resecciones colónicas de urgencia mediante el análisis de la incidencia de dehiscencias de sutura. No obstante, estos estudios no tienen en cuenta el importante sesgo que supone el factor técnico, no pudiendo afirmar por tanto que estas dehiscencias no sean consecuencia de una defectuosa técnica quirúrgica. La mayoría de los trabajos publicados ofrecen cifras de dehiscencias anastomóticas sobre series de pacientes en su gran mayoría no aleatorizadas y randomizadas y sin tener en cuenta en muchos casos factores clínicos como la edad o patología concomitante. De esta forma, es muy difícil extraer conclusiones significativas de series de pa- 
cientes en cuanto a incidencia de dehiscencia de sutura tras la realización de una anastomosis primaria. Es por ello por lo que si se quiere estudiar de qué forma afectan a la sutura intestinal los diferentes factores asociados parece más fiable realizar un modelo experimental animal, donde la técnica quirúrgica es posiblemente un factor más controlado.

La mayoría de los estudios experimentales publicados utilizan para valorar la calidad de la sutura dos técnicas: métodos mecánicos, como es la medición de la tensión de ruptura de la sutura; y métodos de detección del colágeno como el análisis de prolina, hidroxiprolina y/o metaloproteasas o la valoración de las muestras mediante imágenes de microscopía óptica. Los estudios experimentales que utilizan la tensión de ruptura de la sutura como variable directamente asociada al riesgo de fallo de la misma, la miden mediante una sonda de Foley conectada a un manómetro, inflando el balón de la sonda hasta conseguir la dehiscencia de la sutura intestinal (3-5). Este método podría parecer poco fiable y poco sensible para detectar la calidad de la anastomosis, ya que, probablemente, la resistencia de la sutura a la distensión no esté tan directamente relacionada a la dehiscencia de la misma, y, por otro lado, la tensión necesaria para deshacer la sutura realizada en el animal de experimentación, aún controlada mediante manometría, parece una medida sometida a una variabilidad mecánica importante. Mansson y cols. (6) publican un estudio experimental en el que demuestran, con pruebas radiológicas basadas en la extravasación de contraste que la medición de la presión de la anastomosis en ratas no es un método fiable para el estudio de la resistencia de estas suturas.

Otros estudios experimentales utilizan el análisis de prolina y de hidroxiprolina (7), metabolitos del colágeno, para valorar el metabolismo de este, pero estas sustancias no son específicas del colágeno y pueden aparecer en el tejido tras la metabolización de otras moléculas proteicas, por lo que su cuantificación no está relacionada de forma tan directa con las alteraciones producidas en el metabolismo del colágeno.

Savage y Lacombe (8) publican un estudio con conejos en el que evalúa mediante inmunofluorescencia indirecta la presencia de metaloproteasas (MMP), enzimas responsables del catabolismo del colágeno, y del inhibidor tisular de las MMP (TIMP) en la mucosa colónica, relacionando su concentración en la mucosa colónica con la aparición de dehiscencias. La detección de MMP como marcadores del metabolismo del colágeno ha sido utilizada por otros autores en diversos estudios experimentales $(9,10)$. No obstante, las MMP y su inhibidor, son parámetros que se relacionan sólo de forma indirecta con el catabolismo del colágeno.

Otros estudios han utilizado criterios histológicos para evaluar la cicatrización de las anastomosis (11) utilizando una escala basada en grado de tinción de la muestra, celularidad, etc. Es un método que tiene el inconveniente de no ser cuantificable de forma exacta y sometido en gran medida a cierta subjetividad por parte del evaluador.

En el presente trabajo hemos conseguido, mediante técnicas de radioinmunoanálisis, aislar las moléculas de procolágeno (PINP) y de telopéptido terminal del colágeno I (ICTP) como marcadores de la formación y de la degradación, respectivamente, del colágeno I depositado en el tejido colónico, y relacionarlas desde la base de un grupo control, con modelos animales de desnutrición, distensión intestinal y lavado colónico intraoperatorio. Obviamos, por tanto, la incidencia de dehiscencia de la sutura por parecernos un factor con gran dependencia de la técnica quirúrgica, no utilizamos la medición de la tensión de ruptura de la anastomosis medida mediante manometría por considerarla poco sensible respecto a cuantificar la calidad de la sutura, y preferimos no usar la detección de prolina, hidroxiprolina o metaloproteasas, que a nuestro juicio son marcadores menos específicos y con una relación indirecta con la síntesis y la destrucción del colágeno. Las determinaciones de PINP e ICTP, creemos son marcadores de una alta especificidad y sensibilidad, presentando por ello una alta fiabilidad como indicadores del metabolismo del colágeno debido a que son moléculas directamente relacionadas con el mismo.

En el presente estudio utilizamos el colágeno I como marcador de la cicatrización en el tejido colónico por ser el más abundante en esta localización. Estos péptidos se encuentran intactos en el plasma y en la orina directamente después de su liberación del tejido conectivo. En la práctica clínica son marcadores utilizados ampliamente en el estudio de patologías asociadas al metabolismo óseo, ya que se ha comprobado su relación directa con la reabsorción ósea. Su determinación mediante radioinmunoensayo o mediante enzimoinmunoensayo en estos pacientes, es una prueba de rutina desde hace años. Se han publicado además numerosos estudios de estas moléculas en patologías no asociadas al metabolismo óseo, como el cáncer esofágico (12) o el cáncer de mama (13), donde se proponen como marcador pronóstico en el cáncer de tiroides (14), donde pudiera ser marcador del efecto supresor de la levotiroxina, o asociado a tratamientos como la isotretinoina (15). También se han estudiado mediante radioinmunoensayo las modificaciones de colágeno tipo I y III en las placas de aterosclerosis de arterias carótida, femoral común y aorta (1), objetivándose en las mismas una escasa colagenización.

Bode y Karttunen (2), de la Universidad de Oulu en Finlandia, han utilizado la cuantificación del péptido carboxiterminal del colágeno I (ICTP) y III (IIICTP) en muestras de colon para evaluar su concentración en el tejido colónico afecto de cáncer y en el afecto por diverticulosis. El método utilizado en este estudio para la preparación de las muestras titulares en extractos solubles ha sido el utilizado como base para la realización de este trabajo experimental.

Las determinaciones de PINP e ICTP se han analizado directamente sobre el tejido colónico, anastomótico y tejido "sano", por lo que sus variaciones están directamente 
relacionadas con el modelo experimental asociado y su concentración tisular indicativa del metabolismo "in situ" de la molécula colágena y las modificaciones inducidas por cada modelo animal.

Las modificaciones encontradas en la síntesis y destrucción del colágeno de cada grupo de estudio respecto al grupo control A, están relacionadas con la viabilidad de la anastomosis colónica. Es decir, un incremento en la síntesis de colágeno (PINP) o una menor destrucción del mismo (ICTP) implican una mayor viabilidad de la sutura. Por el contrario, un descenso en la síntesis o un incremento en su destrucción incrementan el riesgo de dehiscencia de la misma.

En el tejido colónico del animal sano (grupo A) hemos encontrado unos niveles basales de PINP de 0,434 $\mu$ g por gramo de tejido $(\mu \mathrm{g} / \mathrm{g})$ y unas concentraciones tisulares de ICTP de $0,846 \mu \mathrm{g} / \mathrm{g}$. Al cuarto día de realizar la anastomosis término-terminal, en condiciones anatómicas y metabólicas normales, las concentraciones de PINP son de $0,468 \mu \mathrm{g} / \mathrm{g}$ y los de ICTP de $1,425 \mu \mathrm{g} / \mathrm{g}$. Es decir, existe un incremento de PINP del 7,83\% y un aumento de ICTP del 68,44\% ( $\mathrm{p}=0,000)$. Se comprueba así que la realización de una sutura colónica produce un aumento en el metabolismo del colágeno que se refleja en un incremento estadísticamente significativo tanto en sus niveles de síntesis (PINP) en un 7,83\% como en sus niveles de destrucción (ICTP) en un 68,44\%. Este mayor incremento de ICTP se debe probablemente a que ya se encuentra asentado en la sutura, tras 4 días de cicatrización, el proceso de remodelación habitual de la red colágenica, al mismo tiempo que continúa la síntesis de fibra colágena por parte de las células fibroblásticas.

El modelo experimental de desnutrición se ha conseguido tras 72 horas de ayuno. Para otorgar validez a este grupo de estudio hemos analizado 3 parámetros: pérdida de peso, reducción de concentraciones plasmáticas de albúmina y reducción de la grasa mesentérica.

Después de tres días de ayuno se ha conseguido una reducción media del peso de 350,3 a 325,75 g. Es decir, una disminución de peso del $7 \%(\mathrm{p}=0,032)$, que supone según los estándares establecidos en la práctica clínica, una desnutrición en grado leve (93\% del peso inicial). Asimismo, la media de concentraciones plasmáticas de albúmina obtenidas de sangre arterial tras 72 horas de ayuno son de $982 \mathrm{mg} / \mathrm{dl}$ en el grupo control (A) y 801 $\mathrm{mg} / \mathrm{dl}$ en el grupo de ratas desnutridas (B), o lo que es lo mismo, una diferencia en la concentración plasmática de albúmina del 18,43\% que certifica los hallazgos recogidos en cuanto al peso del animal $(\mathrm{p}=0,000)$. Respecto a la disminución de la grasa mesentérica se trata de un parámetro cualitativo, no cuantitativo, y que, aunque no estaba previsto inicialmente, se concluyó en incluirlo debido a su aparición en todos los animales de este grupo B. Se ha objetivado un descenso cualitativo de la grasa mesentérica en el $100 \%$ de los animales. Estos datos nos hacen afirmar de manera estadísticamente significativa que el modelo animal del grupo B se encuentra en situación de desnutrición proteico-calórica, estableciéndose su validez como grupo de estudio de desnutrición.

Hemos comprobado que la desnutrición reduce los niveles de PINP en el colon de la rata previo a la anastomosis en un 16,59\%, detectándose $0,362 \mu \mathrm{g} / \mathrm{g}$ de PINP en el colon de las ratas desnutridas frente a los $0,434 \mu \mathrm{g} / \mathrm{g}$ recogidos en el grupo control. No sólo eso, sino que en el tejido anastomótico se objetiva también una menor concentración tisular de PINP en el grupo de ratas desnutridas (diferencia del 19,69\%): 0,376 $\mu \mathrm{g} / \mathrm{g}$ de PINP detectados en el grupo B frente a $0,468 \mu \mathrm{g} / \mathrm{g}$ del grupo control. Es decir, el tejido colónico de las ratas desnutridas presenta un depósito menor de colágeno secundario probablemente a una menor capacidad de síntesis en el recambio habitual del colágeno en los tejidos y, además, este hecho se ve reflejado en una reducción en la síntesis de colágeno en la anastomosis.

Hemos detectado unos niveles mayores de ICTP del $12,77 \%$ en el colon de la rata desnutrida de este grupo B respecto al grupo control: 0,954 frente a 0,846 $\mu \mathrm{g} / \mathrm{g}$ en el grupo control. También en la anastomosis son más elevados los niveles de ICTP, hasta $1,640 \mu \mathrm{g} / \mathrm{g}(1,425 \mu \mathrm{g} / \mathrm{g}$ en el grupo control), o lo que es lo mismo, una diferencia de $15,09 \%$. Estos datos, próximos a la significación estadística establecida en el presente estudio, reflejan un incremento en la destrucción del colágeno en los animales desnutridos, tanto en el colon preanastomótico como en la anastomosis del cuarto día, sin poder ofrecer una razón fisiológica adecuada.

Aunque tanto las concentraciones colónicas de PINP como de ICTP se encuentran aumentadas en el tejido anastomótico de las ratas desnutridas en un 3,87 y 71,91\% respectivamente respecto a los niveles detectados en el colon preanastomótico de este grupo, el PINP aumenta menos que en el grupo control $(7,83 \%)(p=0,356)$, no existiendo diferencias respecto al incremento de ICTP.

Todos estos datos apuntan a que existe un descenso estadísticamente significativo de procolágeno (PINP) en el colon y en las anastomosis de las ratas desnutridas respecto al grupo control que sugieren que la desnutrición y la hipoalbuminemia reducen la síntesis de colágeno en las anastomosis intestinales, favoreciendo con ello la aparición de dehiscencias en la sutura. La destrucción del colágeno está incrementada en el colon y en las anastomosis de las ratas desnutridas, aunque no es un dato con significación estadística y no encontramos una razón plausible al respecto.

Es el estado nutricional del paciente uno de los que más se ha estudiado como posible factor de riesgo de aparición de dehiscencias de la sutura intestinal. En este sentido, la albuminemia, y sobre todo, la concentración plasmática de prealbúmina, es el marcador más fiable para detectar posibles déficits nutricionales en los pacientes. La bibliografía consultada aporta estudios referentes al estado clínico y más concretamente, a la desnutrición y la hipoalbuminemia, como factor pronóstico de la viabilidad de una anastomosis primaria (16). Así, Aliev (17) y 
Briskin y Smakov (18), con una serie de 242 y 263 pacientes con cáncer de colon respectivamente, abogan por una descompresión y resolución de los síntomas agudos del paciente tales como la desnutrición, previamente a la intervención quirúrgica como base para reducir la incidencia de fracaso en la anastomosis. Para Koperna y Kisser (19), además de la desnutrición, también la edad avanzada podría influir en el pronóstico de las anastomosis intestinales.

Testini y Margari (20) ya demuestran en un estudio retrospectivo sobre 200 pacientes sometidos a resección colónica y anastomosis término-terminal, que la hipoalbuminemia está asociada a la aparición de fugas anastomóticas. Más amplia es la serie de Longo y Virgo (21), de 4.711 pacientes, en la que demuestran además que el descenso de albúmina es un factor predictivo de mortalidad. Este estudio publicado por Longo y Virgo es, en función de la gran amplitud de la muestra que presenta, significativo desde el punto de vista estadístico en cuanto a demostrar que la hipoalbuminemia incrementa el riesgo de fallos anastomósticos y complicaciones postoperatorias. También Ceriati y cols. (22) propone la hipoalbuminemia como el factor clínico de riesgo más importante de fracaso en las anastomosis intestinales, sólo por detrás de la insuficiencia renal crónica tras estudiar de forma retrospectiva una amplia serie de pacientes intervenidos.

Nuestros resultados experimentales, en los que demostramos que existe una menor síntesis de PINP en las anastomosis de las ratas desnutridas respecto al grupo control, corroboran las conclusiones aportadas por estos autores tras observar que la desnutrición influye de forma negativa sobre la viabilidad de las anastomosis intestinales. Podemos también confirmar el dato que ya expusieron Testini y Margari (20), Longo y Virgo (21) y Ceriati y cols. (22) mediante estudios retrospectivos de series de pacientes describiendo la hipoalbuminemia como marcador pronóstico de fallo de la anastomosis, ya que en nuestro estudio demostramos la reducción de PINP en las anastomosis de las ratas desnutridas con una reducción de la albuminemia del $18,43 \%$. Además, aportamos datos que sugieren que la desnutrición incrementa la destrucción de colágeno en el tejido anastomótico.

Respecto a los estudios experimentales publicados, existen varios artículos referentes a la desnutrición como factor influyente en la viabilidad de las anastomosis intestinales. Ward y cols. (23) publican un trabajo experimental donde comparan 3 grupos de ratas sin alimentación, con alimentación hipoproteica y con dieta normal. Al determinar la presión de ruptura anastomótica en el postoperatorio demuestra una menor presión colónica de ruptura, estadísticamente significativa, en las ratas desnutridas. Law y Ellis (24) publican un estudio experimental con 2 grupos de ratas a las que se someten a nutrición parenteral normal e hipoproteica respectivamente, comprobando como la segunda incide negativamente en la correcta cicatrización tanto de las anastomosis intestinales como de la herida de laparotomía. Delemarre y cols. (25) demuestran mediante la medición de la tensión de ruptura de las anastomosis practicadas en ratas que el aporte nutricional parenteral mejora la resistencia de la sutura. En otro estudio experimental realizado por Domínguez Jiménez y cols. (26) se emplearon 2 grupos de ratas, nutridas y desnutridas, objetivándose una mayor incidencia de dehiscencias de la anastomosis en el grupo de ratas desnutridas, y confirmándose que el estado nutricional afecta la cicatrización colónica y ocasiona inmunodepresión, lo que favorece la formación de abscesos. Kiyama y cols. (27) realizan un estudio experimental donde analiza la tensión de la sutura intestinal en 2 grupos de ratas, uno control y otro con alimentación precoz, concluyendo que en el segundo grupo, la anastomosis es de mejor calidad, incrementando la presión de la sutura respecto al grupo control $(p<0,05)$. Los estudios experimentales presentados correlacionan la concentración plasmática de albúmina con la resistencia de la anastomosis medida mediante manometría o con la presencia de dehiscencias de la sutura, con el consiguiente sesgo que estas técnicas presentan, como ya comentamos en la discusión del método.

Nuestros resultados sugieren, al igual que los trabajos expuestos y mediante un modelo experimental con significación estadística, que la desnutrición favorece la dehiscencia de la sutura intestinal, aunque pensamos que nuestro método de detección PINP e ICTP presenta una mayor fiabilidad que el uso de la tensión de ruptura de la anastomosis utilizada en otros trabajos.

Como conclusiones podemos establecer que: a) el ayuno de 72 horas reduce el peso y la albúmina plasmática; b) la anastomosis colónica incrementa los niveles de procolágeno y de ICTP en el tejido; y c) la desnutrición reduce la colagenización de la anastomosis colónica.

\section{BIBLIOGRAFÍA}

1. Bode MK, Mosorin M. Complete processing of type III collagen in atherosclerotic plaques. Arterioscler Thromb Vasc Biol 1999; 19: 1506-11.

2. Bode MK, Karttunen J. Type I and III collagens in human colon cancer and diverticulosis. Scand J Gastroenterol 2000; 35: 747-52.

3. Ikeuchi D, Onodera H, Aunj T. Correlation of tensile strength with busting presure in the evaluation of intestinal anastomoses. Dig Surg 1999; 16: 478-85.

4. Martínez E, Vázquez A, Larrocha M. Low-residue diets supplemented with fermentable fiber. Effect on experimental colonic anastomosis. Nutr Hosp 1991; 6: 356-63.

5. Sikas N, Imvrios G, Takoudas D, Gakis D, Papanikolaou V.Mycophenolate mofetil impairs the integrity of colonic anastomosis. J Surg Res 2006; 134 (2): 168-72.

6. Mansson P, Zhang XW, Jeppsson B, Thorlacius H. Anastomotic healing in the rat colon: comparison between a radiological method, breaking strength and bursting pressure. Int J Colorectal Dis 2002; 17 : 420-5.

7. Rolando H, Rolandelli MD. Intravenous butyrate and healing of colonic anastomoses in the rat. Dis Colon Rectum 1997; 40: 67-70.

8. Savage FJ, Lacombe DL. Effect of colonic obstruction on the distribution of matrix metalloproteinases during anastomotic healing. Br J Surg 1998; 85: 72-5. 
9. Shaper KR, Savage FJ, Hembry RM. Regulation of matrix metaloproteinases in a model of colonic wound healing in a rabbit. Dis Colon Rectum 2001; 44: 72-82.

10. Agren MS, Andersen TL, Mirastschijski U, Syk I, Schiodt CB, Surve $\mathrm{V}$, et al. Action of matrix metalloproteinases at restricted sites in colon anastomosis repair: an immunohistochemical and biochemical study. Surgery 2006; 140 (1): 72-82.

11. Mutter D, Aprahamian M. Evaluation of human collagen biomaterials in the healing of colonic anastomoses in dogs. Eur J Surg 1997; 163 : 287-95.

12. Nabeya Y. Serum cross-linked I-CTP as a prognostic tumor marker in patients with esophageal squamous cell carcinoma. Cancer 2002; 94 : 940-9.

13. Saarto T, Blomqvist C, Risteli J, Risteli L, Sarna S, Elomaa I. PINP correlates to bone loss and predicts the efficacy of antiresorptive therapy in pre and post.menopausal non-metastatic breast cancer patients. Br J Cancer 1998; 78: 240-5.

14. Toivonen J, Tahtela R, Laitinen K, Risteli K, Valimaki MJ. Markers of bone turnover in patients with differentiated thyroid cancer with and following withdrawal of thyroxine suppresive therapy. Eur J Endocrinol 1998; 138: 667-73.

15. Trifiro G. I-CTP variation in adolescents receiving oral isotretinoin. J Ped-Endocrin Metab 2002; 15: 35-9.

16. Casamayor MC, Gracia JA, Artigas C et al. Oclusión intestinal secundaria a vólvulo de colon transverso. Rev Esp Enferm Dig 2005; 97 : 914-5.

17. Aliev SA. Surgical management in complicated sigmoid cancer. Khirurgiia Mosk 1999; 11: 26-30.
18. Briskin BS, Smakov GM. Occlusive ileus in colonic cancer. Khirurgiia Mosk 1999; 5: 37-40.

19. Koperna T, Kisser M. Emergency surgery for colon cancer in the aged. Arch Surg 1997; 132: 1032-7.

20. Testini M, Margari A. Le deiscenze nelle anastomosi colo-rettali: fattori di rischio. Ann Ital Chir 2000; 71: 433-40.

21. Longo WE, Virgo KS. Risk factors for morbidity and mortality after colectomy for colon cancer. Dis Colon Rectum 2000; 43: 83-91.

22. Ceriati F, Tebala GD, Ceriati E, Coco C, Tebala D, Verbo A, et al. Surgical treatment of left colon malignant emergencies. A new tool for operative risk evaluation. Hepatogastroenterology 2002; 49: 961-6.

23. Ward MWN, Danzi M, Lawin MR, J, Clark CG. The effects of subclinical malnutrition and refeeding on the healing of experimental colonic anastomoses. Br J Surg 1982; 69: 308-10.

24. Law NW, Ellis H. The effect of parenteral nutrition on the healing of abdominal wall wounds and colonic anastomoses in protein-malnourished rats. Surgery 1990; 107: 449-54.

25. Delemarre JB, van de Velde CJ, de Brauw LM, Vree R. Internal biliary drainage, parenteral nutrition and variation in the total parenteral nutrition feeding solutions: influence on the healing of colon anastomosis in jaundiced rats. J Parenter Enteral Nutr 1990; 14: 629-33.

26. Domínguez Jiménez GL, Athié Athié AJ, Mijares García JM, Cárdenas Lailson E, Pérez Reyes E. Efecto de la desnutrición en la anastomosis colónica de la rata. Cirujano General 2001; 23: 81-6.

27. Kiyama T, Onda M, Tokunaga A, Yoshiyuki T, Barbul A. Effect of early postoperative feeding on the healing of colonic anastomoses in the presence of intra-abdominal sepsis in rats. Dis Colon Rectum 2000; 43: S54-8. 